\title{
PYCR1 Promotes Proliferation, Invasion and Drug- Resistance of Lung Cancer Cells by Regulating STAT3-Mediated PI3K/AKT and NF-KB Signaling Pathway
}

\section{Zhanwu Yu}

Department of Thoracic Surgery, Cancer Hospital of China Medical University, Liaoning Cancer Hospital\&Institute

\section{Gebang Wang}

Department of Thoracic Surgery, Cancer Hospital of China Medical University

\section{Chenlei Zhang}

Department of Thoracic Surgery, Cancer Hospital of China Medical University

Yu Liu

Department of Thoracic Surgery, Cancer Hospital of China Medical University

\section{Wei Chen}

Department of Thoracic Surgery, Cancer Hospital of China Medical Unversity

\section{Haoyou Wang}

Department of Thoracic Surgery, Cancer Hospital of China Medicine University

Hongxu Liu ( $\sim$ liuyibob@163.com )

Liaoning Cancer Hospital\&Institute https://orcid.org/0000-0003-0596-3896

\section{Primary research}

Keywords: NSCLS, PYCR1, STAT3, PI3K/AKT pathway, NF-KB pathway

Posted Date: July 1st, 2020

DOI: https://doi.org/10.21203/rs.3.rs-38608/v1

License: (c) (i) This work is licensed under a Creative Commons Attribution 4.0 International License. Read Full License

\section{EDITORIAL NOTE:}


This preprint was withdrawn by Research Square on 11 February 2021 due to concerns with the images. These issues are described on the PubPeer platform. 


\section{Abstract}

Background: Aberrant expression of PYCR1 has been proved to be one of the most pivotal regulators of tumor progress and metastasis. However, the detailed role of PYCR1 in promotion of NSCLC progress is not investigated thoroughly. The present study was aimed to thoroughly investigate the effect of PYCR1 in the growth of NSCLC and the underlying mechanisms, so that provide valuable theoretical basis for efficient treatment of NSCLC.

Methods: The expressions of PYCR1 and its target genes and downstream signals were respectively determined by Western-blot assay and RT-qPCR experiment. Cell growth of NSCLC cells was investigated using the CCK-8 kit while the proliferation assay was performed with the help of EDU staining. The NSCLC-bearing mice mode was established to evaluate the effect of PYCR1 on the progress of NSCLC and the underlying mechanisms in vivo.

Results: It was firstly demonstrated that the PYCR1 was overexpressed in lung cancer tissues and cells and overexpression of PYCR1 contributed to significantly enhanced proliferation, invasion, and drugresistance of cancer cells and progress of NSCLC tissues. Further studies revealed that up-regulation of PYCR1 level resulted to the elevation of bioactivity of STAT3. Additionally, the positive correlation between the expression of PYCR1 and STAT3 indicated that the PYCR1 promotes the growth of NSCLC through targeting regulation the STAT3 levels. Furthermore, activation of STAT3 by PYCR1 was proved to be able of awaking the PI3K/AKT and NF-KB signaling pathway, which was extremely important to the metabolism, proliferation, cell survival, and growth of many cancer cell types.

Conclusion: In conclusion, our study demonstrated that PYCR1 was specifically overexpressed in lung cancer and closely related to rapid progress and drug-resistance thorough regulating the STAT3-mediated $\mathrm{PI} 3 \mathrm{~K} / \mathrm{AKT}$ and NF-KB signaling pathway. It may provide a valuable strategy for treatment of malignant lung cancer.

\section{Background}

As one of the most malignant tumors, lung cancer was generally characterized with fast growth rate, high morbidity, and terrible mortality [1,2]. According to the statistics, the morbidity and mortality of lung cancer ranks first among men [3]. The non-small cell lung cancer (NSCLC) represents one of the most aggressive type of lung cancer, which accounts for $85 \%$ all mortality cases globally $[4,5]$. Treatment of NSCLC are still seriously blocked by the rapid metastasis, fast progress, and insufficient therapy approaches [6]. Additionally, majority of patients with lung cancer will develop multi-drug resistance in the later stage, resulting in insensitive to chemotherapy and high mortality $[7,8]$.

Pyrroline-5-carboxylate reductase 1 (PYCR1), an enzyme involved in cell metabolism, has been shown to be up-regulated in many cancer types [9-12]. Previous study has proved that knockdown of PYCR1 significantly inhibited the proliferation, colony, and invasion of prostate cancer cells [9]. In addition, over expression of PYCR1 was detected in breast cancer and down-regulation of PYCR1 levels markedly 
decrease the proliferation ability of breast cancer cells, while obviously enhanced the apoptosis rate of cancer cells [10]. Importantly, high level of PYCR1 was also detectable in NSCLC and it was high expression of PYCR1 closely related to the progression of NSCLC [11]. However, the aberrant expression of PYCR1 in NSCLC and its role and mechanisms in regulating progress of lung cancer are still need to be thoroughly investigated, so as to provide a new theoretical basis for the diagnosis and treatment of lung cancer.

The signal transducer and activator of transcription protein (STAT), belongs to the family of cytoplasmic transcription factors, has been demonstrated played significant role in the signal transduction through multiple mechanisms [13]. Increasing evidences revealed that the STAT3 was involved in the development and progression of many cancer types through participation in a series of tumorigenic processes such as cell proliferation and survival [14]. Moreover, aberrant expression and constitutive activation of STAT3 have been detected in a variety of tumors such as the gastric cancer, breast cancer, prostate tumor, and NSCLC [13-15]. Additionally, knockdown of STAT3 was proved to be facilitate to enhance the chemotherapy efficacy of malignant cancer by inhibition cell proliferation, invasion, and inhibition of drug-resistance [16].

The present study was aim to explore the effect of PYCR1 on proliferation, invasion and drug resistance of lung cancer cells and the underlying mechanisms. Additionally, the mutual relation between the PYCR1 and STAT3 was further investigated.

\section{Materials And Methods}

\section{Chemicals and reagents}

The cell counting kit-8 (CCK-8) was purchased from Dojindo Molecular Technologies (Gaithersburg, MD). 5-Fluorouracil was obtained from Aladdin (Shanghai, China). Primary antibodies and horseradish peroxidase (HRP)-conjugated secondary antibodies were purchased from Santa Cruz Biotechnology (Santa Cruz, CA). The complete RPMI-1640 medium supplemented with 10\% fetal bovine serum (FBS), $100 \mathrm{U} / \mathrm{ml}$ penicillin and $100 \mu \mathrm{g} / \mathrm{ml}$ streptomycin was purchased from Hyclone (GE Healthcare Life Sciences, Logan, UT, USA).The EdU kit was obtained from Guangzhou RiboBio Co., Ltd. (Guangzhou, China) and the Hoechst 33342 was purchased from Molecular Probes (Eugene, OR, USA).

\section{Cell culture and animals}

The human lung adenocarcinoma cell lines $\mathrm{H} 520, \mathrm{H} 1299, \mathrm{H} 1975$, and $\mathrm{A} 549$ cells were achieved from the Chinese Academy of Medical Sciences (Shanghai, China) while the normal cell line BEAS-2B was obtained from the American Type Culture Collection (ATCC, Rockville). All the cells were cultured in the RPMI- 1640 medium as mentioned above under a $5 \% \mathrm{CO}_{2}$ atmosphere at $37^{\circ} \mathrm{C}$. The male nude mice with weight of $20 \pm 2 \mathrm{~g}$ and average of $4 \sim 5$ weeks were obtained from BK Lab Animal Ltd. (Shanghai, China).

\section{Cell transfection}


For cell experiments, A549 cells were transfected respectively in advance with various siRNAs (GenePharma, Shanghai, China) including the Ctrl-siRNA, si-PYCR1, p-PYCR1, and STAT3. The cell transfections were performed using the Lipofectamine 2000 (Invitrogen, NY, USA) with the help of plasmid vectors pcDNA3.1. Finally, the transfection efficiencies were evaluated after $48 \mathrm{~h}$ of the transfection using the Western-blot assay.

\section{Cell proliferation and invasion}

Cells were seeded in 96-well plates at the density of $5 \times 10^{3}$ cells/well. After an overnight of incubation, the old medium was replaced with fresh cell culture medium and continue to culture for $24 \mathrm{~h}$. Then 50 $\mathrm{mM}$ EdU was added and co-incubated with cells for $2 \mathrm{~h}$ followed by fixation with $4 \%$ formaldehyde for 15 min. After that, the cells were incubated with 1X Apollo reaction cocktail and stained with Hoechst 33342 for $30 \mathrm{~min}$. Finally, the results were visualized by the fluorescence microscope (Carl Zeiss).

To investigate the invasion ability, cells were seeded on the upper chamber of trans-well at the density of $1 \times 10^{5}$ cells/well. The lower compartment was filled with $00 \mathrm{~mL}$ of complete medium containing $20 \%$ FBS. Then the cells were allowed to incubate for $24 \mathrm{~h}$. After that, the non-invading cells on the upper chamber were removed while the invaded cells were fixed by $4 \%$ paraformaldehyde. For quantitative analysis, the cells were stained with hematoxylin followed by counting cell numbers using Image $\mathrm{J}$ software.

\section{CCK-8 assay}

$5 \times 10^{3}$ cells were seeded in 96 -well plates and cultured at $37^{\circ} \mathrm{C}$. After treated as indicated, the cells were allowed to grow for 24 . Then $10 \mu \mathrm{L}$ CCK-8 solution was added into each well of the plates. After incubation for $4 \mathrm{~h}$, the absorbance was determined by microplate reader (BioTek Instruments, Winooski, VT) with the detection wavelength was set at $450 \mathrm{~nm}$.

\section{Western blotting analysis}

The radioimmunoprecipitation assay lysis buffer (Cell Signaling Technology, Inc.) was applied to extract the total protein of cell samples. Then the protein concentration was determined using the BCA Protein Assay kit (Pierce Biotechnology, Inc., Rockford, IL, USA). For separation of the protein samples, $10 \%$ SDSPAGE (Sigma-Aldrich) was applied and the gel was allowed to run for 20 min under $80 \mathrm{~V}$ followed by 100 min under $120 \mathrm{~V}$. After that, the samples were transferred to a polyvinylidene fluoride membrane (EMD Millipore, Billerica, MA, USA) followed by blocking with $5 \% \mathrm{w} / \mathrm{v}$ non-fat dry milk (Sigma-Aldrich). For protein detection, corresponding primary antibodies were introduced and incubated with protein samples for overnight at $4^{\circ} \mathrm{C}$. HRP-labeled secondary antibodies was subsequently added and allowed to incubate with samples for $1 \mathrm{~h}$ under room temperature. Finally, an enhanced chemiluminescence kit (KPL, Inc., Gaithersburg, MD, USA) was used to determine the immunobands followed by analysis with ImageJ (version 1.48; National Institutes of Health, Bethesda, MD, USA) software. 


\section{RT-qPCR experiments}

The total RNA in cells and tumor tissues was extracted using the Trizol reagent (Invitrogen, Carlsbad, CA). After the concentration of RNA was determined, the reverse transcription (RT) reaction was performed by the PrimeScript RT Master Mix (Perfect Real Time; Takara Bio Inc., Tokyo, Japan). Subsequently, the PCR reaction was performed thorough the ABI PRISM 7900 Real-Time system (Applied Biosystems, Foster City, CA). Finally, the $2^{-\Delta \Delta C t}$ method was applied to determine the levels of various RNA with all RNA molecules were normalized to the GAPDH. The primers were adopted here were displayed as follows: PYCR1 forward, ACACCCCACAACAAGGAGAC, and reverse, CTGGAGTGTTGGTCATGCAG; PI3K forward, CATCACTTCCTCCTGCTCTAT, and reverse, CAGTTGTTGGCAATCTTCTTC; AKT forward, GGACAACCGCCATCCAGACT, and reverse, GCCAGGGACACCTCCATCTC; NF-kB forward, GCCGTGGAGTACGACAA, and reverse, CGGTTTCCCATTTAGTATGT; MMP-1 forward, AAATAGTGGCCCAGTGGTTG, and reverse, CACATCAGGCACTCCACATC; VEGF forward, TGTCTAATGCCCTGGAGCCT, and reverse, TTAACTCAAGCTGCCTCGCC; P-gp forward, CCCATCATTGCAATAGCAGG, and reverse, GTTCAAACTTCTGCTCCTGA; GAPDH forward TGTGTCCGTCGTGGATCTGA, and reverse, CCTGCTTCACCACCTTCTTGA.

\section{Immunofluorescence staining}

Cells were fixed with $4 \%$ paraformaldehyde for 10 min and then washed with PBS before incubated with 0.5\% Triton-20 (Sigma-Aldrich) for $10 \mathrm{~min}$. After that, the samples were blocked with $4 \%$ bovine serum albumin (Sigma-Aldrich) for $1 \mathrm{~h}$ under room temperature. Then primary antibodies were added and incubated with samples for overnight at $4^{\circ} \mathrm{C}$ followed by incubated with fluorochrome-conjugated secondary antibody for $1 \mathrm{~h}$ at room temperature. The results were analyzed under a confocal laser scanning microscopy (Leica TCS SP5II; Leica Microsystems GmbH, Wetzlar, Germany).

\section{Immunohistochemistry (IHC) assay}

The tumor tissues were sectioned for $5-\mu \mathrm{m}$ thick and then fixed with $4 \%$ paraformaldehyde for $10 \mathrm{~min}$. After they were blocked with $4 \%$ bovine serum albumin, primary antibodies was introduced and coincubated with the samples for overnight at $4^{\circ} \mathrm{C}$. To visualize the target proteins, the horse radish peroxidase (HRP)-labeled secondary antibodies was allowed to react with the samples for $1 \mathrm{~h}$. Subsequently, the sections were stained with 3,3'-diaminobenzidine for $10 \mathrm{~min}$ and counterstained with $0.5 \%$ hematoxylin for $1 \mathrm{~min}$ before evaluation under the confocal laser scanning microscopy.

\section{Statistical analysis}

Results in the study were presented as means \pm standard error of the mean (SEM) using GraphPad Prism 7 software. Two and one-way analysis of variance (ANOVA) were used for compare between different treatments with $p$-values $<0.05$ considered significant, $<0.01$ very significant and $<0.001$ highly significant. 


\section{Results}

\section{Overexpression of PYCR1 was detected in lung cancer tissues and cell lines}

As determined by the RT-qPCR and Western-blot assay, the expression levels of PYCR1 in lung cancer tissues was significantly higher than that of the normal adjacent tissues (Figure 1A and B). Such results were further confirmed by the cellular PYCR1 expression. As shown in Figure 1C and 1D, the normal BEAS-2B cells expressed the lowest level of PYCR1. In contrast, obvious higher levels of PYCR1 was detected in the lung cancer cell lines, including the H520, H1299, H1975, and A549 cells. Of great importance, the drug-resistant A549 cells expressed the strongest signal of PYCR1. In this case, the A549 cells were selected as the cell model for further experiments.

\section{Transfection with si-PYCR1 significantly inhibited the proliferation, invasion, and drug resistances of A549 cells}

Firstly, the transfection efficiency was determined with results shown in Figure 2A revealed that cells transfection with si-PYCR1 leaded to obvious down-regulation of PYCR1 expression in A549 cells. However, negligible changes were determined in the cells transfected with Ctrl-siRNA.

As shown in Figure 2B, silencing the PYCR1 expression leaded to an obvious down-regulated proliferation ability of A549 cells. Further investigation revealed that knockdown of PYCR1 resulted in dramatically decreased expression of vascular endothelial growth factor (VEGF) (Figure 2C), the primary genes regulating the proliferation of cells. Then the vision ability of A549 cells was evaluated after various treatments. Results displayed that the invasion rate of A549 cells was markedly inhibited after transfection with si-PYCR1 by decreasing the levels of matrix metalloproteinase-1 (MMP-1), the main genes regulating cell invasion (Figure 2D and 2E). Whether silencing of PYCR1 could alleviate the drugresistance of malignant lung cancer cells was subsequently determined. As shown in Figure $2 \mathrm{~F}$, downregulation of PYCR1 levels significantly enhanced the chemotherapy effect of 5-Fu, a well-known anticancer agent. Additionally, further investigation exhibited that the expression of P-gp, the most important drug-resistant related protein, was dramatically down-regulated in A549 cells after transfection with siPYCR1.

\section{Overexpression of PYCR1 dramatically enhanced the proliferation, invasion, and drug resistances of A549 cells}

Determination of the transfection efficiency revealed that the significantly higher expression of PYCR1 was detected in the A549 cells transfection with p-PYCR1 while not the Vector (Figure 3A). Further studies exhibited that high levels of PYCR1 was quite helpful in the proliferation of A549 cells thorough upregulation of the VEGF expression (Figure 3B and 3C). Moreover, increase of PYCR1 expression also facilitated the invasion of A549 cells by elevation of the MMP-1 levels (Figure 3D and 3E). In contrast to the above results, transfection of A549 cells with p-PYCR1 significantly increased the $\mathrm{IC}_{50}$ value of 5 -FU and up-regulated the expression of P-glycoprotein (P-gp). 


\section{PYCR1 enhanced cell progress thorough targeting the STAT3}

As shown in Figure 4A, the binding site between the STAT3 and PYCR1 was predicated by the ChIPBase. Subsequently, the binding of PYCR1 with STAT3 was further confirmed using the CoIP experiments. The results clearly illustrated that similar expression trend was obtained between the PYCR1 and STAT3. For further confirmation, the Western-blot assay and RT-qPCR assay was conducted to determine the levels of STAT3 after treating cells with p-PYCR1. Interestingly, transfection the lung cancer cells with p-PYCR1 dramatically increased the level of STAT3 (Figure 4B). Spearman's analysis further revealed that the expression of STAT3 was liner correlation to the PYCR1 expression. Taking these results together, it could be concluded that the STAT3 was the targeting gene of PYCR1.

\section{PYCR1 promoted the proliferation, invasion, and drug resistances of A549 cells thorough regulating the STAT3 expression}

As shown in Figure 5A, proliferation assay revealed that transfection of A549 cells with STAT3 significantly enhanced the proliferation ability. However, the proliferation rate of A549 cells was signally down-regulated by transfection with si-PYCR1. Further investigation displayed that transfection of cells with STAT3 leaded to obvious increase of VEGF expression while treated with si-PYCR1 resulted in contrary results (Figure 5B). Invasion assay showed that the STAT3 treated A549 cells have the strongest invasion rate by increase of MMP-1 expression (Figure 5C and 5D). Interestingly, such favorable effect could be signally discounted by additional transfection with si-PYCR1, suggested that the si-PYCR1was against the expression of STAT3. IC ${ }_{50}$ values in Figure 5E exhibited that high expression of STAT3 contributed to an adverse effect on the chemotherapy efficacy of 5-Fluorouracil (5-Fu), which was mainly because that overexpression of STAT3 significantly increase the P-gp level in A549 cells (Figure 5F).

Finally, qualitative analysis of various proteins in A549 cells after various treatment was determined. The results shown in Figure $5 \mathrm{G}$ exhibited the similar trend to above with transfection with STAT3 significantly enhanced the expression of VEGF, MMP-1, and P-gp.

\section{PYCR1 promoted the activation of STAT3-mediated PI3K/AKT and NF-KB Signaling Pathway}

As shown in Figure 6A, overexpression of STAT3 leaded to high expression of PI3K, p-PI3K, AKT, and pAKT, suggested that high level of STAT3 contributed to the activation of PI3K/AKT signaling pathway. Additionally, transfection with STAT3 also resulted in up-regulation of NF-KB p65 and p-p65, indicated that the NF-KB signaling pathway could also be activated by STAT3. For confirmation, RT-qPCR experiments were performed. As demonstrated in Figure 6B-6D, the levels of p-P13K/PI3K, p-AKT/AKT, and p-p65/p65 were signally elevated after transfection with STAT3. Moreover, the immunofluorescence analysis obtained the similar results as above with the stat 3 treated cells achieved the highest levels of p65 and AKT (Figure 6E).

PYCR1 promoted lung tumor growth thorough the activation of STAT3-mediated PI3K/AKT and NF-KB Signaling Pathway 
Tumor images in Figure 7A and quantitative analysis in Figure 7B showed that the PYCR1 treated mice exhibited the largest tumor volumes while contrary result was obtained by si-PYCR1 transfection. Immunohistochemical analysis revealed that treatment of PYCR1 leaded to high expression of VEGF, MMP-1, and P-gp in tumor tissues (Figure 7C), indicated that the PYCR1 promoted lung cancer growth thorough enhancing cancer cells proliferation, invasion, and drug-resistance. Quantitative analysis by RTqPCR displayed that overexpression of PYCR1 contributed to high expression of p-P13K/PI3K (Figure 7D), p-AKT/AKT (Figure 7E), and p-p65/p65 (Figure 7F) in lung cancer tissues. Such results could be further confirmed by qualitative evaluation via Western-blot assay (Figure 7G).

\section{Discussion}

Lung cancer represents one of the most commonly diagnosed cancer with rapid progress, high metastatic, and high mortality [1-3]. Despite the development of a wide range of novel therapeutic methods, the treatment efficacy of lung cancer remain dissatisfactory with and overall 5-year survival rate was only $10 \%$ around $[2,4]$. It have been demonstrated that a wide array of proteins were involved in the tumorigenesis, progress, metastasis, and drug-resistance of lung cancer [17-19]. Among those molecules, PYCR1 represents one of the most seductive proteins because overexpression of PYCR1 was suggested to be the most related factor that contributed to poor outcomes of patients with malignant cancer [11-13]. In the present study, we demonstrated that the levels of PYCR1 was significantly higher in lung cancer tissues and cells than of the tumor adjacent normal tissues and normal cells.

It has been previously demonstrated that aberrant activation of PYCR1 was detected in a wide array of cancer types, including colorectal cancer [20], prostate cancer [21], and breast cancer [22]. Additionally, the aberrant expression of PYCR1 significantly influenced the tumor size, grade, subtypes, and even that survival of patient with malignant cancer [23]. Recent studies revealed that high level of PYCR1 was facilitated to tumor cells proliferation and depletion of PYCR1 signally suppressed the competence of tumor growth and invasion $[23,24]$. Our study demonstrated that high expression of PYCR1 was exactly quite helpful in the proliferation and invasion of lung cancer cells thorough increase the VEGF and MMP1 levels, respectively. Additionally, up-regulation of PYCR1 in lung cancer cells was closely related to drugresistance by dramatically increased the level of P-gp expression. In contrast, silencing the PYCR1 expression in lung cancer cells resulted in signally down-regulated ability of proliferation, invasion and drug-resistance of cancer cells.

As a transcription factor, STAT3 has been detected with overexpression and/or hyperactivation in multiple cancer types [25]. Thorough transcription of anti-apoptotic and pro-proliferative genes, STAT3 played significantly role in the enhancement of cell proliferation, invasion, and drug-resistance of cancer cells [25]. Additionally, it was also demonstrated that STAT3 was able of direct interaction with mitochondrial DNA to facilitate the Ras-dependent malignant transformation and cancer progression [25-27]. Of great importance, the aberrant expression of STAT3 was suggested to be one of the most important factor that associated with poor overall survival rates in patients with malignant cancer [27]. Our study revealed that elevation of STAT3 expression significantly enhanced the proliferation ability of lung cancer cells. More 
importantly, it was demonstrated that the STAT3 could be activated by PYCR1 and the level of STAT3 in lung cancer was almost linear correlation to the expression of PYCR1. It was exhibited that up-regulation of PYCR1 in cells resulted in significant increase of STAT3 expression. In contrast, decrease of PYCR1 level by treating cells with si-PYCR1 dramatically down-regulated the STAT3 expression, which finally leaded to insufficient proliferation, invasion and more sensitivity to chemotherapy.

Phosphatidylinsitol-3-kinase (PI3K)-protein kinase B (AKT) pathway has been recommended as one of the key intracellular signal transduction pathway that contributed to promotion of metabolism, proliferation, cell survival, and invasion of cancer cells [28-30]. Previous studies demonstrated that the $\mathrm{PI3K} /$ Akt pathway was directly related to multiple functions of cancer cells, such as the quiescence, proliferation, and cancer progress $[28,29]$. In the present study, high level of STAT3, resulted from transfection of cells or treating tumor-bearing mice with PYCR1, contributed to high expression of PI3K, p$\mathrm{PI} 3 \mathrm{~K}, \mathrm{AKT}$, and $\mathrm{p}-\mathrm{AK} T$. Therefore, it could be concluded that aberrant expression of PYCR1 in lung cancer cells or tissues was facilitated to the activation of PI3K/AKT signaling pathway. Additionally, PI3K and AKT can regulate the activation of the NF-KB pathway through the phosphorylation of $\mathrm{IKBa}$ [31]. In this case, blocking of the PI3K/AKT pathway and NF-KB was supposed to be a favourable option for cancer treatment [32]. Our further experiments revealed that high expression of STAT3 also contributed to the upregulation of NF-KB p65 and p-p65, suggested that NF-KB signaling pathway was activated after treating by PYCR1. It was further demonstrated that the levels of p-P13K/PI3K, p-AKT/AKT, and p-p65/p65 could be signally elevated by transfection with PYCR1. Taking these together, our study confirmed that the PYCR1 promoted the activation of STAT3-mediated PI3K/AKT and NF-KB signaling pathway.

\section{Conclusion}

In conclusion, the present study confirmed that high levels of PYCR1 was detectable in A549 cells and overexpression of PYCR1 contributed to promotion of cancer cell proliferation, invasion, and drugresistance. Importantly, our studies further revealed that PYCR1 promoted progress of NSCLS thorough targeting the STAT3-mediated PI3K/AKT and NF-KB signaling pathway. The findings suggested that inhibition PYCR1 and targeting PI3K/AKT and NF-KB signaling pathway may be a valuable strategy for treatment of NSCLS.

\section{Abbreviations}

NSCLC, Non-small cell lung cancer; PYCR1, Pyrroline-5-carboxylate reductase 1; STAT3, Signal transducer and activator of transcription 3; FBS, fetal bovine serum; VEGF, Vascular endothelial growth factor; MMP1, Matrix metalloproteinase; P-gp, P-glycoprotein; 5-FU, 5-Fluorouracil; PI3K/AKT, Phosphatidylinsitol-3kinase (PI3K)-protein kinase B (AKT), IHC, Immunohistochemistry; HRP, horse radish peroxidase.

\section{Declarations}

\section{Authors' contributions}


Zhanwu Yu, Gebang Wang, and Hongxu Liu were responsible for the guarantor of integrity of the entire study and preparation, editing, and review of the manuscript. Chenlei Zhang, Yu Liu, and Wei Chen were respectively involved in the definition of intellectual content and literature research. Wei Chen and Haoyou Wang responsible for the data acquisition and analysis. Hongxu Liu revised the manuscript.

\section{Funding}

The present research is supported by the General Program of Natural Science Foundation of Liaoning Province (2015020464), Major Science and Technology Platform of Liaoning Higher Institution (No.2916009), Science Research Foundation for Absorbed Talents Program of Liaoning Cancer Hospital, and Special Project of Applied Technology of Population and Health by Shenyang Science and Technology Plan (No.18-014-4-04).

\section{Acknowledgement}

We thank the supports from all participants.

\section{Availability of data and materials}

The data are available from the authors upon request.

\section{Ethics approval and consent to participate}

The animal experiments in the present study were all performed in accordance with guidelines evaluated and approved by the ethics committee of Cancer Hospital of China Medical University.

\section{Consent for publication}

Not applicable.

\section{Competing interests}

The authors declare that they have no competing interests.

\section{References}

1. Balboni TA, Hui KP, Kamal AH. Supportive Care in Lung Cancer: Improving Value in the Era of Modern Therapies. Am Soc Clin Oncol Educ Book. 2018;38:716-25.

2. Schveigert D, Krasauskas A, Didziapetriene J, et al. Smoking, hormonal factors and molecular markers in female lung cancer. Neoplasma. 2018;63:504-9.

3. Soufi $M$, Arimura $H$, Nagami N. Identification of optimal mother wavelets in survival prediction of lung cancer patients using wavelet decomposition-based radiomic features. Med Phys. 2018;45:5116-28. 
4. Laohathai S, Cho S, Yum S, Jang HJ, Seong YW, Kim K, Jheon S. Clinical and functional outcomes after curative resection in octogenarians with clinical stage I non-small cell lung cancer. J Geriatr Oncol. 2019;10:436-8.

5. Tominaga T, Tsuchiya T, Mochinaga K, Arai J, Yamasaki N, Matsumoto K, Miyazaki T, Nagasaki T, Nanashima A, Tsukamoto K, Nagayasu T. Epidermal growth factor signals regulate dihydropyrimidine dehydrogenase expression in EGFR-mutated non-small-cell lung cancer. BMC Cancer. 2016;16:354.

6. Li J, Guo NN, Jin HR, Yu H, Wang P, Xu GG. Effects of exercise training on patients with lung cancer who underwent lung resection: a meta-analysis. World J Surg Oncol. 2017;15:158.

7. Chen X, Zhou JY, Zhao J, Chen JJ, Ma SN, Zhou JY. Crizotinib overcomes hepatocyte growth factormediated resistance to gefitinib in EGFR-mutant non-small-cell lung cancer cells. Anticancer Drugs. 2013;24:1039-46.

8. Zhang H, Hu B, Wang Z, Zhang F, Wei H, Li L. miR-181c contributes to cisplatin resistance in nonsmall cell lung cancer cells by targeting Wnt inhibition factor 1. Cancer Chemother Pharmacol. 2017;80:973-84.

9. Ye Y, Wu Y, Wang J. Pyrroline-5-carboxylate reductase 1 promotes cell proliferation via inhibiting apoptosis in human malignant melanoma. Cancer Manag Res. 2018;10:6399-407.

10. Ding J, Kuo ML, Su L, Xue L, Luh F, Zhang H, Wang J, Lin TG, Zhang K, Chu P, Zheng S, Liu X, Yen Y. Human mitochondrial pyrroline-5-carboxylate reductase 1 promotes invasiveness and impacts survival in breast cancers. Carcinogenesis. 2017;38:519-31.

11. Sang S, Zhang C, Shan J. Pyrroline-5-Carboxylate Reductase 1 Accelerates the Migration and Invasion of Nonsmall Cell Lung Cancer In Vitro. Cancer Biother Radiopharm. 2019;34:380-7.

12. Zhuang J, Song Y, Ye Y, He S, Ma X, Zhang M, Ni J, Wang J, Xia W. PYCR1 interference inhibits cell growth and survival via c-Jun N-terminal kinase/insulin receptor substrate 1 (JNK/IRS1) pathway in hepatocellular cancer. J Transl Med. 2019;17:343.

13. Ioza V, Tamauskaite T, Vilkeviciute A, Pasvenskaite A, Liutkevicius V, Liutkeviciene R. Determination of SIRT1 rs12778366, FGFR2 rs2981582, STAT3 rs744166, and RAGE rs1800625 Single Gene Polymorphisms in Patients with Laryngeal Squamous Cell Carcinoma. Dis Markers 2019:3907232.

14. Jiang X, Wu M, Xu Z, Wang H, Wang H, Yu X, Li Z, Teng L. HJC0152, a novel STAT3 inhibitor with promising anti-tumor effect in gastric cancer. Cancer Manag Res. 2018;10:6857-67.

15. Gong QZ, Xiao D, Gong GY, Xu J, Wen XD, Feng F, Qu W. EH-42: A Novel Small Molecule Induces Apoptosis and Inhibits Migration and Invasion of Human Hepatoma Cells through Suppressing STAT3 Signaling Pathway. Curr Cancer Drug Targets. 2019;19:583-93.

16. Fang L, Gao L, Xie L, Xiao G. GC7 enhances cisplatin sensitivity via STAT3 signaling pathway inhibition and elF5A2 inactivation in mesenchymal phenotype oral cancer cells. Oncol Rep. 2018;39:1283-91.

17. Abu Hejleh T, Furqan M, Ballas Z, Clamon G. The clinical significance of soluble PD-1 and PD-L1 in lung cancer. Crit Rev Oncol Hematol. 2019;143:148-52. 
18. Zakaria N, Yusoff NM, Zakaria Z, Lim MN, Baharuddin PJ, Fakiruddin KS, Yahaya B. Human nonsmall cell lung cancer expresses putative cancer stem cell markers and exhibits the transcriptomic profile of multipotent cells. BMC Cancer. 2015;15:84.

19. Luo T, Wang L, Wu P, Gong W, Chen W, Zhao H, Zheng Z. Downregulated vimentin and upregulated Ecadherin in T1 stage non-small-cell lung cancer: does it suggest a mesenchymal-epithelial. transition? Neoplasma. 2017;64:693-9.

20. Roth U, Razawi H, Hommer J, Engelmann K, Schwientek T, Müller S, Baldus SE, Patsos G, Corfield AP, Paraskeva C, Hanisch FG. Differential expression proteomics of human colorectal cancer based on a syngeneic cellular model for the progression of adenoma to carcinoma. Proteomics. 2010;10:194202.

21. Zeng T, Zhu L, Liao M, Zhuo W, Yang S, Wu W, Wang D. Knockdown of PYCR1 inhibits cell proliferation and colony formation via cell cycle arrest and apoptosis in prostate cancer. Med Oncol. 2017;34:27.

22. Sun T, Song Y, Yu H, Luo X. Identification of IncRNA TRPM2-AS/miR-140-3p/PYCR1 axis's proliferates and anti-apoptotic effect on breast cancer using co-expression network analysis. Cancer Biol Ther. 2019;20:760-73.

23. Ding J, Kuo ML, Su L, Xue L, Luh F, Zhang H, Wang J, Lin TG, Zhang K, Chu P, Zheng S, Liu X, Yen Y. Human mitochondrial pyrroline-5-carboxylate reductase 1 promotes invasiveness and impacts survival in breast cancers. Carcinogenesis. 2017;38:519-31.

24. Cai F, Miao Y, Liu C, Wu T, Shen S, Su X, Shi Y. Pyrroline-5-carboxylate reductase 1 promotes proliferation and inhibits apoptosis in non-small cell lung cancer. Oncol Lett 20018;15:731-740.

25. Huynh J, Chand A, Gough D, Ernst M. Therapeutically exploiting STAT3 activity in cancer - using tissue repair as a road map. Nat Rev Cancer. 2019;19:82-96.

26. Gough DJ, Corlett A, Schlessinger K, Wegrzyn J, Larner AC, Levy DE. Mitochondrial STAT3 supports Ras-dependent oncogenic transformation. Science. 2009;324:1713-6.

27. Gough DJ, Marié IJ, Lobry C, Aifantis I, Levy DE. STAT3 supports experimental K-RasG12D-induced murine myeloproliferative neoplasms dependent on serine phosphorylation. Blood. 2014;124:225261.

28. Wang HJ, Yang ZX, Dai XT, Chen YF, Yang HP, Zhou XD. Bisdemethoxycurcumin sensitizes cisplatinresistant lung cancer cells to chemotherapy by inhibition of CA916798 and PI3K/AKT signaling. Apoptosis. 2017;22:1157-68.

29. Li Y, Ma X, Wang Y, Li. miR-489 inhibits proliferation, cell cycle progression and induces apoptosis of glioma cells via targeting SPIN1-mediated PI3K/AKT pathway. Biomed Pharmacother. 2017;93:43543.

30. Shariati M, Meric-Bernstam F. Targeting AKT for cancer therapy. Expert Opin Investig Drugs. 2019;28:977-88.

31. Zhu J, Wen K. Astragaloside IV inhibits TGF- $\beta 1$-induced epithelial-mesenchymal transition through inhibition of the PI3K/Akt/NF-KB pathway in gastric cancer cells. Phytother Res. 2018;32:1289-96. 
32. Liu W, Wang S, Sun Q, Yang Z, Liu M, Tang H. DCLK1 promotes epithelial-mesenchymal transition via the PI3K/Akt/NF-KB pathway in colorectal cancer. Int J Cancer. 2018;142:2068-79.

\section{Figures}

A

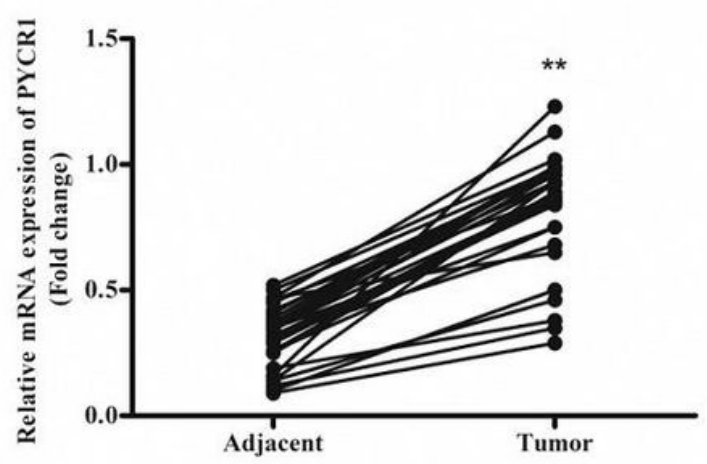

C

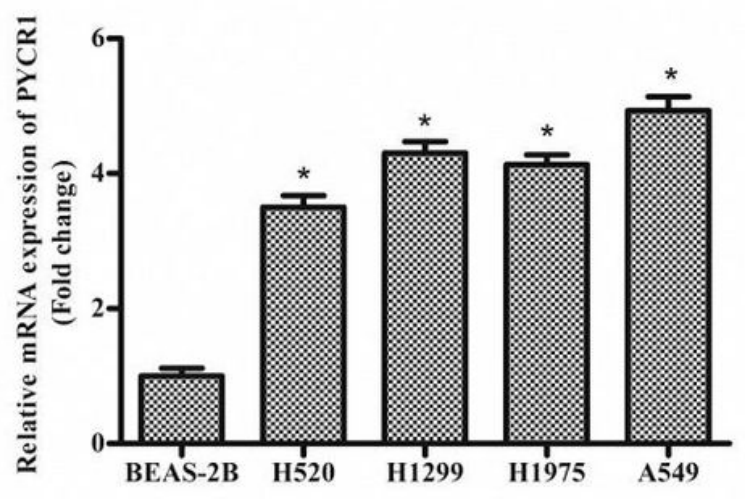

B

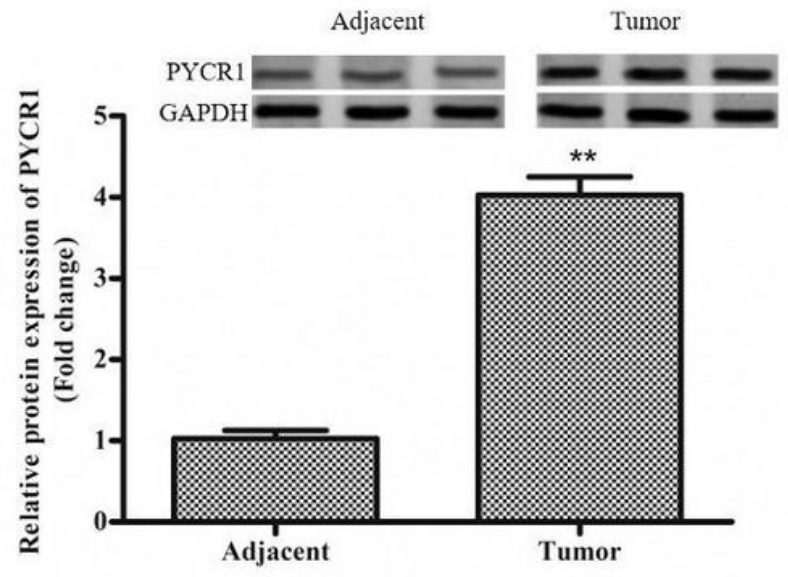

D

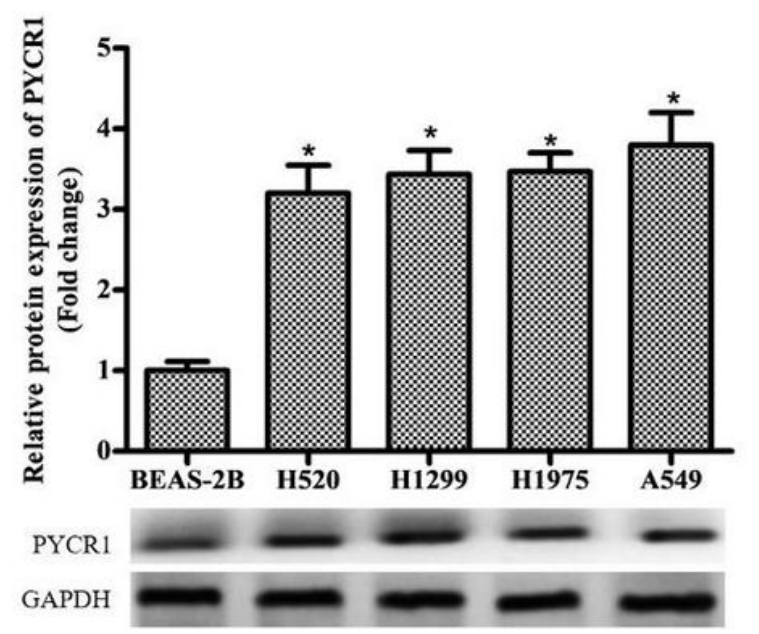

Figure 1

PYCR1 expression levels in lung tumors and cells, and respectively compared with that in tumor adjacent normal tissues and normal cell lines. (A) Expression of PYCR1 in lung cancers and para-carcinoma tissues determined by the RT-qPCR experiments. (B) Qualitative and semi-quantitative analysis of the PYCR1 expression levels in lung tumor and adjacent normal tissues by Western-blot assay. (C) Cellular expression of PYCR1 analyzed in various lung cancer cell lines and compared with the normal cells. (D) Qualitative and semi-quantitative analysis of PYCR1 expression in lung cancer cells and normal cells via the Western-blot assay. All experiments were performed repeatedly three times. ${ }^{*} P<0.05,{ }^{\star \star} P<0.01$, significantly higher than the tumor adjacent normal tissues or BEAS-2B cells. 
A

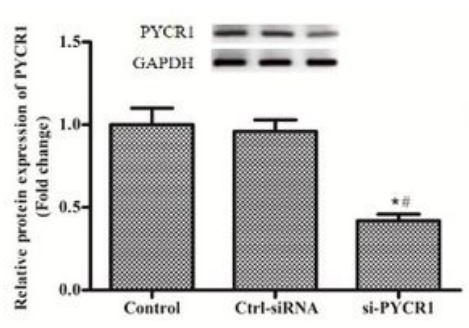

B

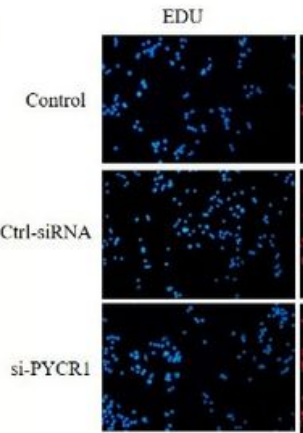

BRDU

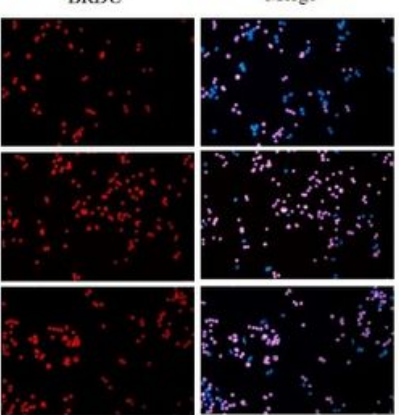

C

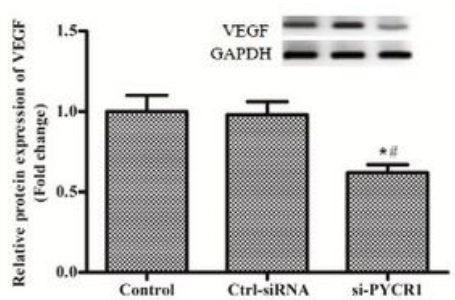

D

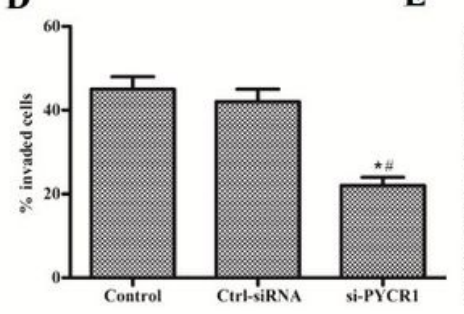

$\mathbf{E}$

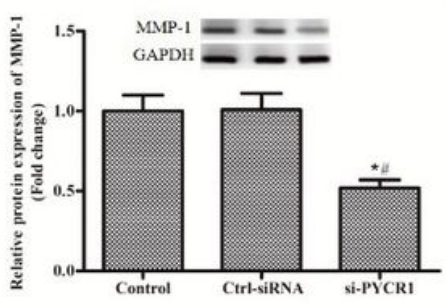

F

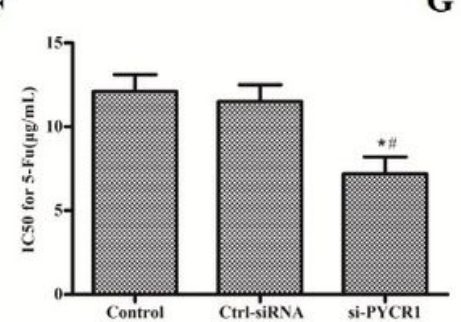

G

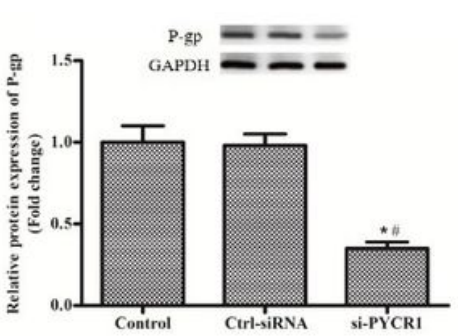

Figure 2

Silencing the PYCR1 expression significantly inhibited the proliferation, invasion, and drug resistances of A549 cells. (A) Transfection efficacy of ctrol-siRNA and si-PYCR1 determined by Wester-blot assay. (B) Proliferation of A549 cells after transfected with ctrol-siRNA and si-PYCR1 determined using the immunocytochemical analysis. (C) Investigation of the VEGF level in the ctrol-siRNA and si-PYCR1 transfected A549 cells. (D) Invasion rate of A549 cells after respectively treated by ctrol-siRNA and siPYCR1. (E) MMP-1 levels in the ctrol-siRNA and si-PYCR1 transfected A549 cells. (F) IC50 values of 5-Fu on A549 cells treated by ctrol-siRNA or si-PYCR1, and compared with the untreated cells. (G) P-gp expression in the ctrol-siRNA and si-PYCR1 transfected A549 cells. All experiments were performed repeatedly three times. ${ }^{*} \mathrm{P}<0.01$, significantly higher than the control group. 
A

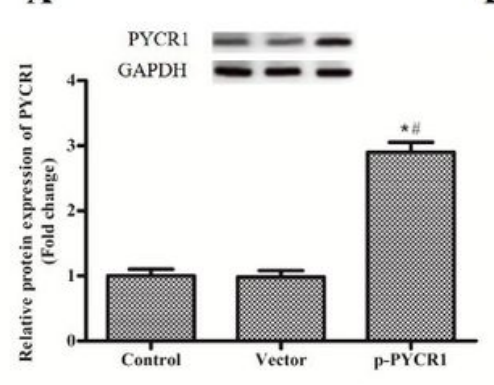

B
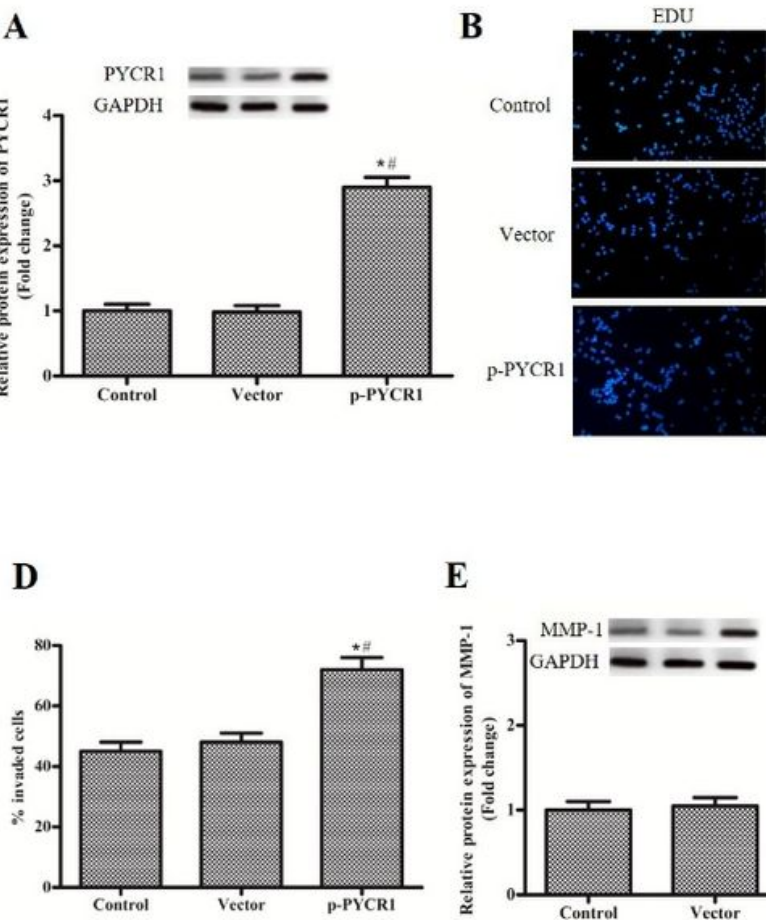

$\mathbf{E}$

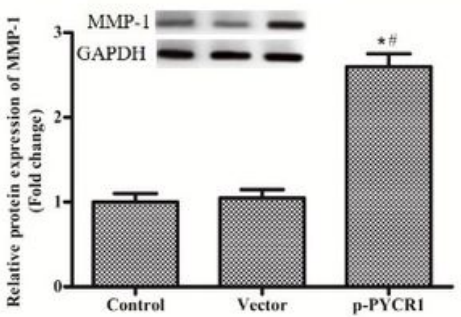

BRDU

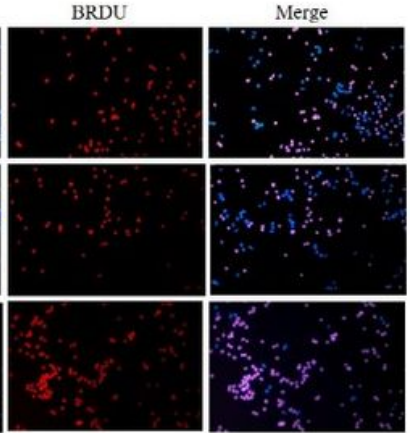

$\mathbf{F}$

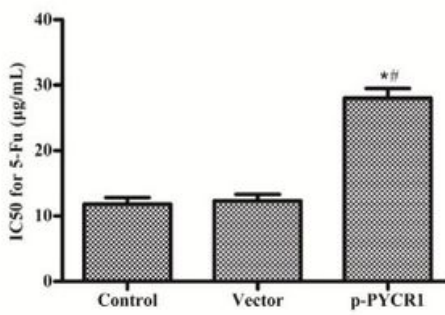

C

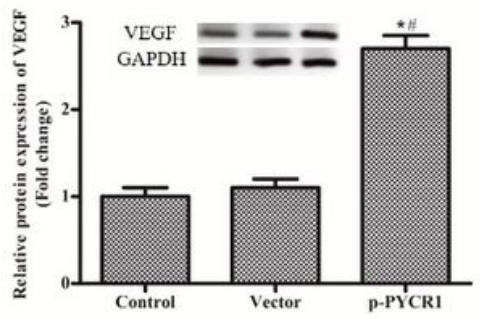

Figure 3

Overexpression of PYCR1 significantly promoted the proliferation, invasion, and drug resistances of A549 cells. (A) Transfection efficacy of vector and p-PYCR1 determined by Wester-blot assay. (B) Proliferation of A549 cells after transfected with vector and p-PYCR1 determined using the immunocytochemical analysis. (C) Investigation of the VEGF level in the vector and p-PYCR1 transfected A549 cells. (D) Invasion rate of A549 cells after respectively treated by vector and p-PYCR1. (E) MMP-1 levels in the vector and p-PYCR1 transfected A549 cells. (F) IC50 values of 5-Fu on A549 cells treated by vector and pPYCR1, and compared with the untreated cells. (G) P-gp expression in the vector and p-PYCR1 transfected A549 cells. All experiments were performed repeatedly three times. ${ }^{*} \mathrm{P}<0.01$, significantly higher than the control group. 
A

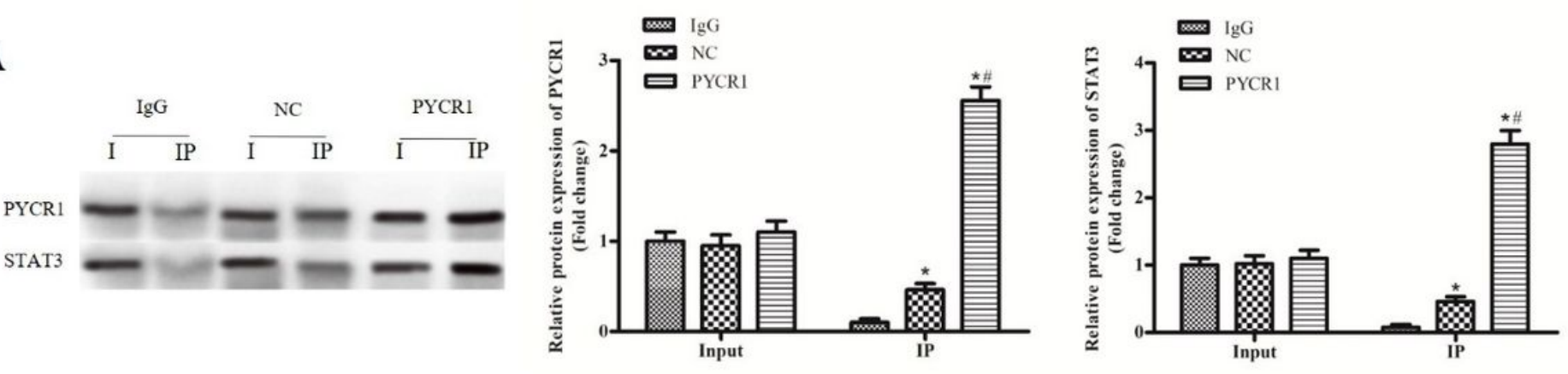

B

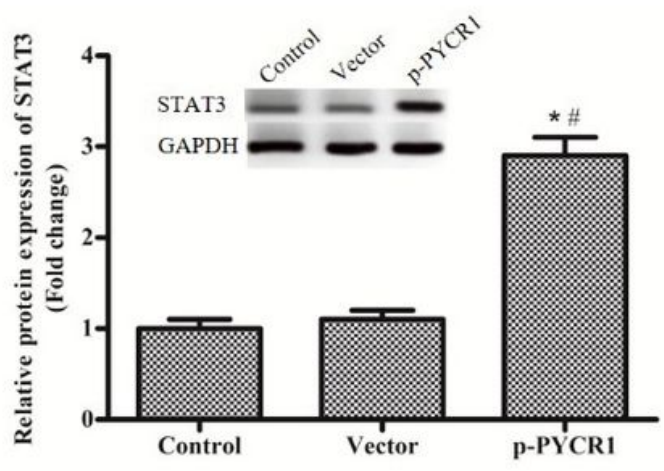

C

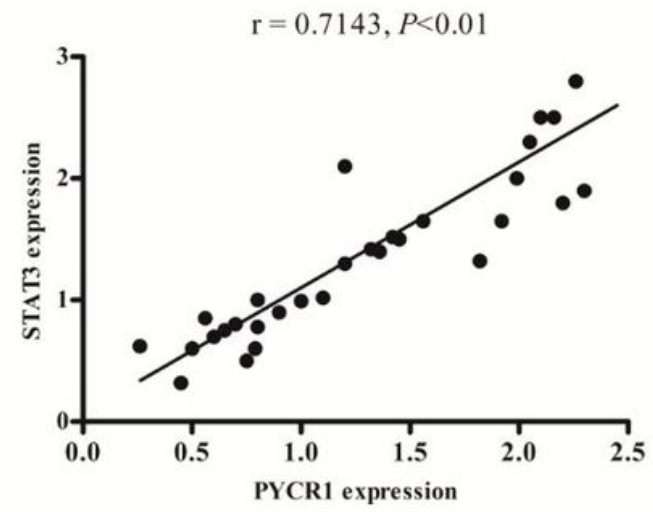

\section{Figure 4}

Overexpression of PYCR1 significantly up-regulated the level of STAT3 in lung cancer cells. (A) The binding site between the STAT3 and PYCR1 was predicated by the ChIPBase and further confirmed using the CoIP experiments. (B) The levels of STAT3 after treating cells with p-PYCR1 was detected by Westernblot assay. (C) Spearman's analysis of the expression correlation between the STAT3 and PYCR1. * $\mathrm{P}<0.05, \# \mathrm{P}<0.05$, significantly higher than the control group. 


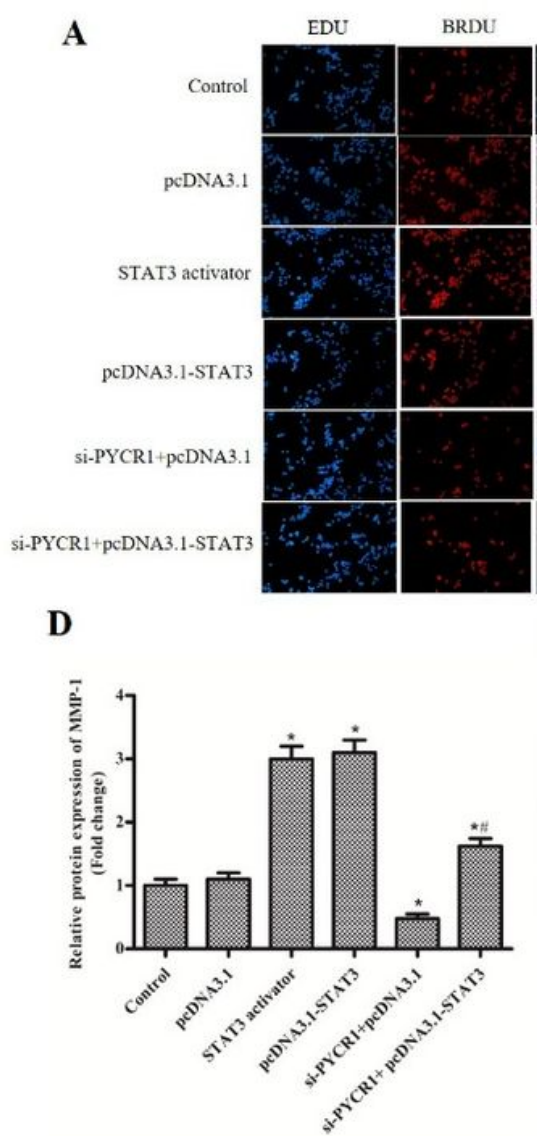

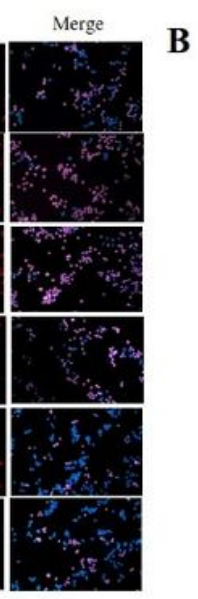

E

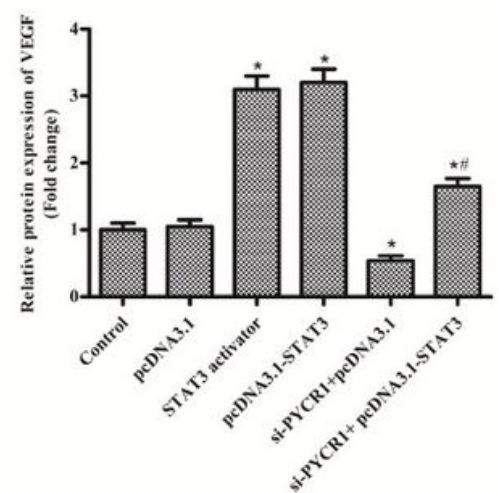

F
C

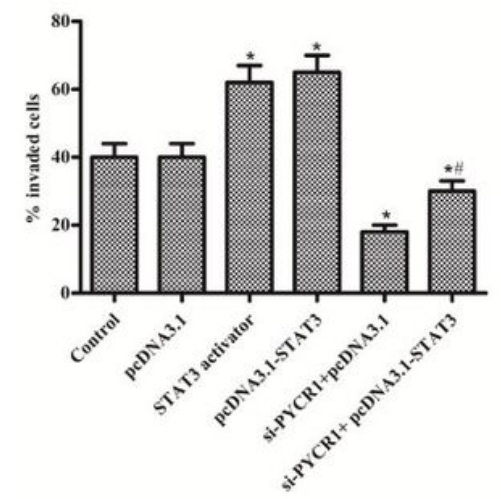

G

VEGH VEGF

P-gp

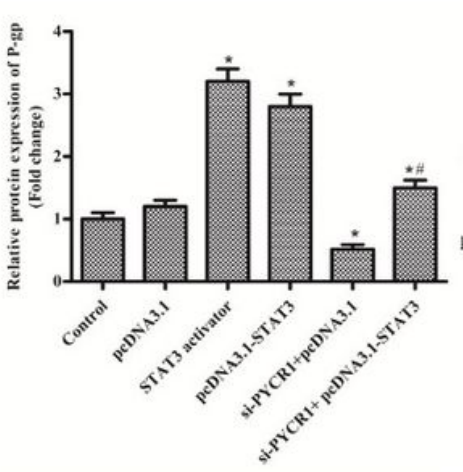
T3 activator si-PYCRI peDNA3.1-STAT3

Figure 5

PYCR1 promoted the proliferation, invasion, and drug resistances of A549 cells thorough regulating the STAT3 expression. (A) Proliferation of A549 cells after transfected with various genes. (B) Determination of VEGF levels in various genes transfected cells by RT-qPCR. (C) Invasion rated of of A549 cells after transfected with various genes. (D Determination of MMP-1 levels in various genes transfected cells by RT-qPCR. (E) IC50 values of A549 cells after transfected with various genes. (F) Determination of P-gp levels in various genes transfected cells by RT-qPCR. (G) Qualitative analysis of VEGF, MMP-1, and P-gp levels in various genes transfected cells by Western-blot assay. All experiments were performed repeatedly three times. ${ }^{*} \mathrm{P}<0.05,{ }^{\star}{ }^{*} \mathrm{P}<0.01$, significantly higher than the control group. 
A

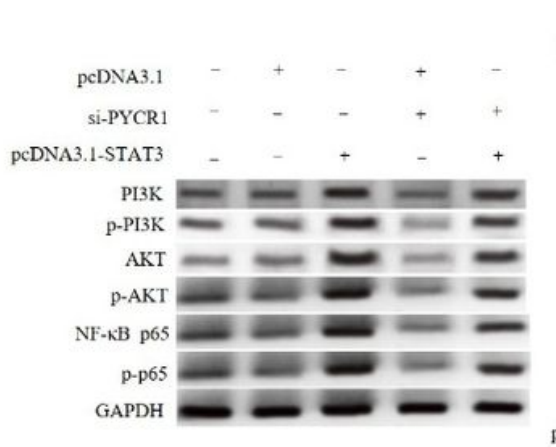

D

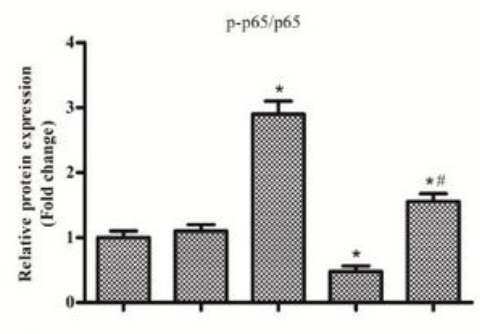

pCDNA3.1 si-PYCR1 peDNA3.1-STAT
B

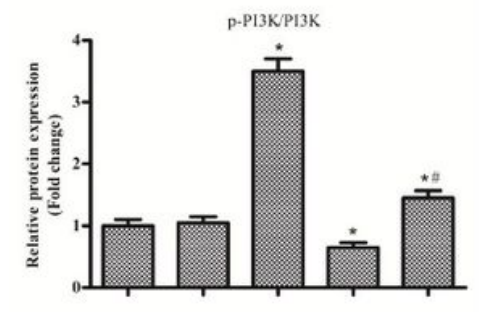

pcDNA3.1

si-PYCR1

pcDNA3.1-STAT3

$\mathbf{E}$

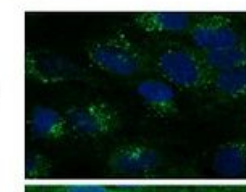

AKT

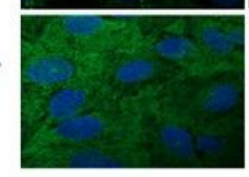

Control

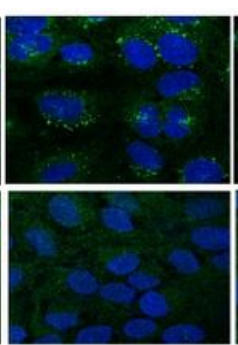

pcDNA3.1
C

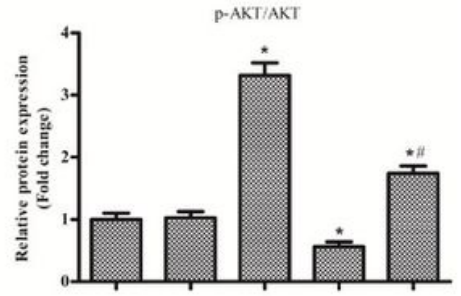

pCDNA3.1

Si-PYCR1

pcDNA3.I-STAT3

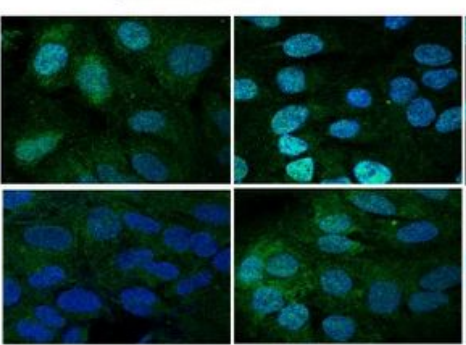

pcDNA3.1-STAT3

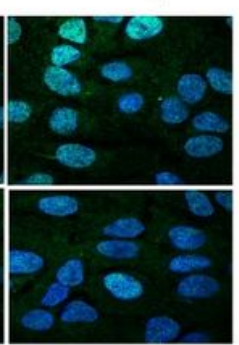

si-PYCR1+ pcDNA3.1 si-PYCR1+ pcDNA3.1-STAT3

Figure 6

PYCR1 promoted the activation of STAT3-mediated PI3K/AKT and NF-KB Signaling Pathway. (A) Qualitative analysis of the PI3K/AKT and NF-KB signaling pathway related genes in A549 cells after various treatment by Western-blot assay. Quantitative investigation of the levels of $p-P 13 K / P I 3 K(B), p-$ AKT/AKT (C), and p-p65/p65 (D) in various genes treated A549 cells by RT-qPCR experiments. (E) Immunofluorescence analysis of p65 and AKT in A549 cells after various treatment. All experiments were performed repeatedly three times. ${ }^{*} \mathrm{P}<0.05,{ }^{*} \mathrm{P}<0.01$, significantly higher than the control group. 
A

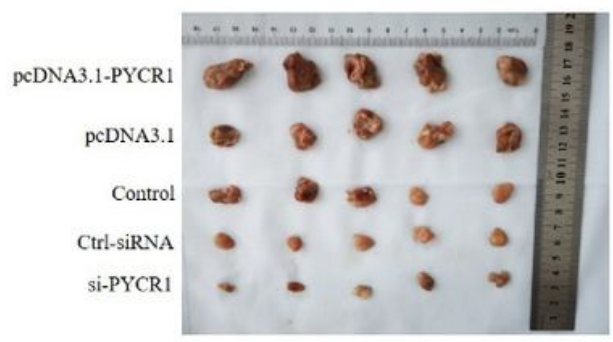

B

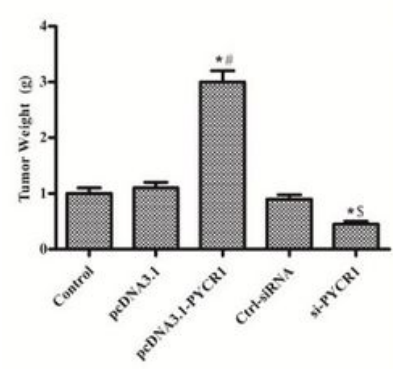

E

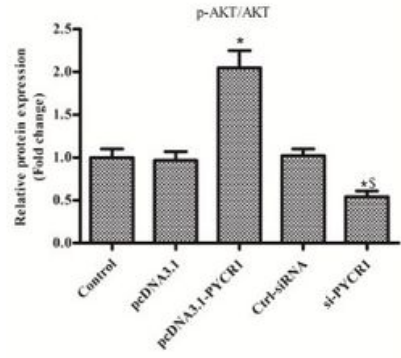

D

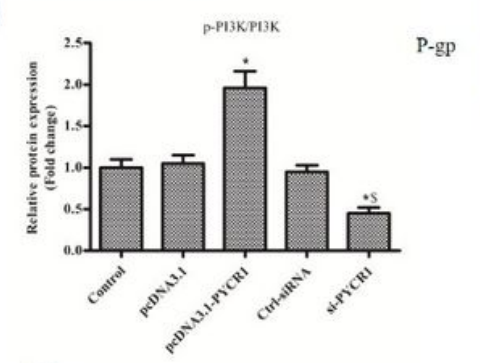

$\mathbf{F}$

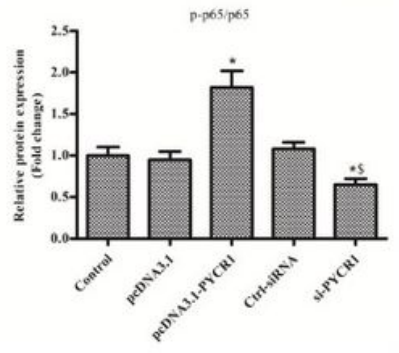

C

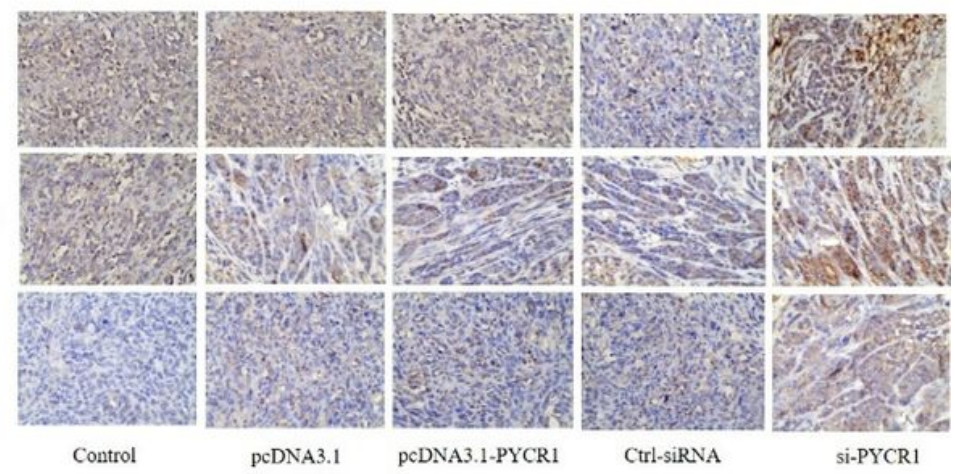

G

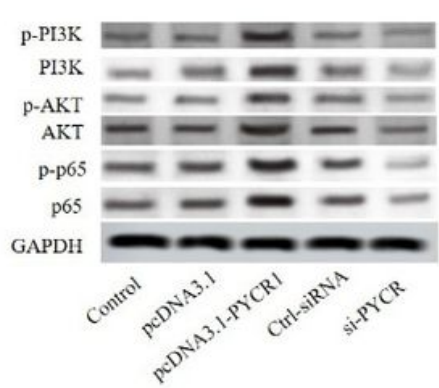

Figure 7

PYCR1 promoted lung tumor growth thorough the activation of STAT3-mediated PI3K/AKT and NF-KB Signaling Pathway. (A) Tumor images of lung cancer after treated by various strategies. (B) Tumor weights of lung cacerafter various treatments. (C) Immunohistochemical analysis of VEGF, MMP-1, and P-gp levels in tumor tissues post transfection with different genes. Quantitative investigation of the levels of p-P13K/PI3K (D), p-AKT/AKT (E), and p-p65/p65 (F) in various genes treated tumor tissues by RT-qPCR experiments. (G) Qualitative analysis of the PI3K/AKT and NF-KB signaling pathway related genes in tumor tissues after various treatment by Western-blot assay. ${ }^{*} P<0.05,{ }^{*} \mathrm{P}<0.01$, significantly higher than the control group.

\section{Supplementary Files}

This is a list of supplementary files associated with this preprint. Click to download.

- CoverLetter.docx 